\title{
LAS REINVINDICACIONES DE LA PATENTE DE INVENCIÓN
}

Alberto Bercovitz

\section{Significado y función de las reivindicaciones}

La patente otorga a su titular un derecho exclusivo de explotación sobre la invención que constituye su objeto.

Por tanto, para determinar los derechos del titular y, en sentido contrario, aquello que en virtud de la patente está prohibido a los terceros, es preciso delimitar el objeto de la patente y delimitarlo de la manera más precisa posible, tanto en interés del titular como de los terceros.

Históricamente la delimitación del objeto de la patente se hacía, en una primera etapa, en base a la descripción, deduciendo de ella lo que constituía la invención protegida y objeto del derecho exclusivo de explotación.

Este sistema, que pervivió en Francia hasta la ley de 1968, plantea, sin embargo, importantes problemas, por la inseguridad jurídica que genera, además de las corruptelas a que da lugar. Se decía, en efecto, que cuanto menos precisa fuera la descripción, más extensa era la protección de la patente.

Esa inseguridad para el titular de la patente $y$, en particular, para los terceros, es la que ha hecho que hoy día, en las legislaciones más avanzadas, se exija que la solicitud de patente incluya las reivindicaciones que delimitan lo que ha de ser protegido como objeto de la patente.

La práctica de delimitar lo que es objeto de la patente por medio de reivindicaciones procede de los sistemas anglosajones. En el Reino Unido ya existía esa práctica en el siglo XVIII, y la ley norteamericana de 1836 
fue la primera que exigió su inclusión en la solicitud. En el Derecho alemán fue la ley de 1891 la que introdujo la exigencia de las reivindicaciones.

Hoy día esa exigencia se ha generalizado. Buena muestra de ello son los artículos 4, 6 y 11 del Tratado de Cooperación en Materia de Patentes (PCT), firmado en Washington el 19 de junio de 1970 (con diversas modificaciones posteriores), y 80 y 84 del Convenio sobre Concesión de Patentes Europeas, hecho en Munich el 5 de octubre de 1973 (citado en adelante como CPE).

El artículo $84 \mathrm{CPE}$-que se corresponde literalmente con el artículo 6 PCT- establece lo siguiente: «Las reivindicaciones definen el objeto para el que se solicita la protección. Deben ser claras y concisas y han de fundarse en la descripcion»».

Así pues, las reivindicaciones definen y delimitan el objeto de la patente. Son, podría decirse, el corazón mismo, el núcleo esencial, de la patente. Cuando la ley se refiere al objeto de la patente, se refiere a ese objeto tal como queda delimitado por las reivindicaciones.

Su función se explica perfectamente por comparación con lo que ocurre con la propiedad de los bienes inmuebles.

En efecto, en los bienes inmuebles hay que señalar con exactitud los linderos, para determinar con claridad el objeto de la propiedad y, en definitiva, los límites que deben respetar los terceros.

En la patente las reivindicaciones vienen a marcar los «linderos» del objeto sobre el que recae el derecho exclusivo y que los terceros han de respetar igualmente.

Lo que ocurre es que la redacción de las reivindicaciones, en cuanto tienen que definir la invención que se protege, es ciertamente más difícil que el deslinde de una finca, que se hace por simple referencia a elementos materiales preexistentes (un camino, un río, una vía férrea, un bosque, etc...).

Por tanto, las reivindicaciones sirven para delimitar de manera precisa cuál es el objeto protegido por la patente, esto es, el objeto de la patente. 
Por ello, las reivindicaciones son el elemento más importante de la patente, y esto se refleja a todos los efectos del Derecho de patentes.

En primer lugar, no existe jurídicamente una solicitud de patente, si no tiene reivindicaciones. Una solicitud que no tenga reivindicaciones no atribuye ninguna fecha de prioridad (art. $80 \mathrm{CPE}$ ).

Durante el procedimiento de concesión, si existe informe sobre estado de la técnica, ese informe tiene, en parte, la función de que el solicitante, a la vista del mismo, pueda modificar sus reivindicaciones (Regla 86.1 del Reglamento de ejecución del CPE y artículo 36.2 de la Ley española de patentes, de 20 de marzo de 1986, citada en adelante como LP).

En los sistemas de concesión con examen previo de la novedad y actividad inventiva, el examen sirve también para delimitar de la forma más adecuada posible las reivindicaciones, en colaboración con el examinador (art. 97.2 a CPE).

Pero, en cualquier caso, la responsabilidad de la redacción de las reivindicaciones es del solicitante. Cuando existe examen previo, la Administración puede denegar la patente si el solicitante no acepta la redacción propuesta, pero no puede imponerle una redacción.

Al delimitar las reivindicaciones el objeto de la patente, ello significa que la exigencia de los requisitos de patentabilidad ha de referirse al objeto de la patente tal como está delimitado por las reivindicaciones, tanto para el procedimiento de concesión, como para la eventual nulidad.

Y, del mismo modo, el objeto del derecho exclusivo otorgado por la patente viene delimitado también por las reivindicaciones. Éstas delimitan el ámbito objetivo de protección de la patente, esto es, la regla técnica cuya explotación corresponde al titular de la patente en exclusiva.

Obsérvese, y esto es muy importante, que lo que aparece en la descripción, pero no es reivindicado, no está protegido.

Hay que señalar, sin embargo, que la prioridad unionista no se vincula estrictamente a las reivindicaciones de la solicitud inicial (art. $4 \mathrm{H}$ del Convenio de la Unión de París para la Protección de la Propiedad Industrial, 
texto de Estocolmo de 14 de julio de 1967, citado en adelante como CUP). Será preciso que la solicitud inicial en la que se basa esa prioridad reúna los requisitos necesarios para obtener fecha de presentación, entre los que estará normalmente que la solicitud incluya una o varias reivindicaciones. Pero para disfrutar de la prioridad unionista basta que el conjunto de los documentos de la solicitud originaria revele los elementos para los que se invoca la prioridad unionista.

\section{Requisitos de fondo}

Según señala MOUSSERON, los requisitos de fondo de las reivindicaciones son tres:

— Deben determinar suficientemente el objeto reivindicado, esto es, la invención para la que se pide protección.

- Deben tener apoyo suficiente en la descripción.

- Deben respetar la exigencia de la unidad de invención.

A continuación se va a hacer referencia a cada uno de estos requisitos.

\section{Determinación del objeto reivindicado}

Las reivindicaciones deben definir claramente la invención para la que se pide protección y que ha de constituir el objeto de la patente. Recuérdese, en efecto, que las reivindicaciones «definen el objeto para el que se solicita la protección» (arts. 84 CPE y 6 PCT).

Esa definición debe ser de aquello que ha sido inventado, que no estaba en el estado de la técnica, y que, por tanto, se trata de proteger.

La definición debe hacerse por las características técnicas de la invención, no por sus aspectos comerciales, y, como es lógico, debe incluir todas las características necesarias para que la invención quede perfectamente delimitada. 
Es decir, que por la lectura de la reivindicación sola, los terceros deben poder conocer la regla técnica que está protegida, sin necesidad de tener que acudir a la descripción o los dibujos de la solicitud.

Debe ser, pues, una definición suficiente en sí misma, lo que no excluye, naturalmente, que haya que recurrir a la descripción y los dibujos para que el tercero pueda saber cómo se ejecuta la invención definida en las reivindicaciones.

$Y$ es que para los terceros es fundamental, por razones de seguridad jurídica, que por la simple lectura de las reivindicaciones se pueda saber en qué consiste la regla técnica cuya explotación está reservada en exclusiva al titular de la patente.

\section{Apoyo en la descripción}

La finalidad básica de la patente es hacer pública la invención que constituye su objeto, describiéndola de tal manera que cualquier experto en la materia pueda ejecutarla. Por ello se exige que la solicitud contenga una descripción suficiente de la invención.

Para conseguir y estimular esa descripción es para lo que se concede el derecho exclusivo de explotación sobre la invención descrita. Y para que los terceros puedan conocer claramente el ámbito del derecho exclusivo, es para lo que se impone al solicitante la redacción de las reivindicaciones.

Así pues, la descripción y las reivindicaciones cumplen funciones diversas, pero complementarias e intímamente ligadas. La descripción relata lo que debe hacer un experto para poder ejecutar la invención; y las reivindicaciones delimitan y definen la invención para que los terceros sepan qué es aquello sobre lo que recae la exclusiva del titular de la patente.

Pero es obvio que la descripción tiene que exponer la forma de ejecutar precisamente la invención que se define en las reivindicaciones, o, dicho de otro modo, que la invención que se reivindica debe estar suficientemente descrita para que cualquier experto pueda ejecutarla.

Esta vinculación entre descripción y reivindicaciones, que viene im- 
puesta por la función misma de la patente, es la que se manifiesta en los textos legales al exigir que las reivindicaciones «han de fundarse en la descripción» (arts. 84 CPE y 6 PCT). Sería, en efecto, absurdo que pudieran reivindicarse válidamente invenciones no descritas.

En definitiva, esto significa que para que lo reivindicado quede realmente protegido es preciso que, además, esté descrito. Del mismo modo que lo descrito sólo quedará protegido por la patente, si además figura en las reivindicaciones.

Si se reivindica lo que no está descrito, normalmente existirá una insuficiencia de la descripción, que es causa de nulidad de la patente (art. 138.1.b CPE).

En los sistemas con examen previo, dentro del propio procedimiento administrativo se rechazarán las reivindicaciones que no tengan un apoyo suficiente en la descripción.

Por supuesto, el hecho de que las reivindicaciones tengan que tener un fundamento suficiente en la descripción, no significa que reivindicaciones y descripción tengan que coincidir exactamente. Esa coincidencia total no es necesaria, porque, como se ha expuesto, ambos elementos cumplen funciones diversas.

En la descripción, el solicitante expondrá pormenorizadamente todo lo que hay que hacer para ejecutar la invención; expondrá las actuaciones concretas que han de efectuarse y los experimentos que justifican la eficacia de la regla inventiva.

Se tratará siempre de actuaciones y experimentos concretos.

Pero en las reivindicaciones, no se trata de dar indicaciones concretas sobre la forma de ejecutar la invención, sino que se trata de definir en abstracto esa invención, de la forma más clara y más general posible.

Por eso se dice que la elaboración de las reivindicaciones, partiendö de la descripción, supone un proceso de generalización a una delimitación más abstracta de la regla inventiva cuya ejecución en casos concretos se ha expuesto en la descripción, esto es, la generalización de uno o más ejemplos particulares. 
La fundamentación de las reivindicaciones en la descripción es suficiente, si partiendo de la información contenida en ésta, un experto en la materia fuera capaz de extender la enseñanza contenida en la descripción para hacerla corresponder con el objeto cuya protección se reivindica, recurriendo a métodos de experimentación o análisis habituales. Así lo establecen las directivas de la Oficina Europea de Patentes (enero de 1992 - Parte C, Cap. III, $n^{\circ}$ 6).

Naturalmente el nivel de generalización admisible habrá que juzgarlo caso por caso, teniendo en cuenta el sector técnico al que la invención se refiere y el nivel técnico alcanzado anteriormente en ese mismo sector.

Así, por ejemplo, la generalización es más necesaria en la química, donde las invenciones pueden referirse a familias de millares o miles de sustancias, que en el campo de la mecánica.

En otro aspecto, si una invención abre un campo totalmente nuevo, es admisible que la reivindicación se redacte en términos más generales que en los casos en que la invención supone un progreso, pero dentro de la técnica ya conocida. Así se señala también en las directivas de la Oficina Europea de Patentes.

Cuando las reivindicaciones implican una generalización que no tiene base en lo descrito, falta la fundamentación exigible en la descripción.

Las directivas de la Oficina Europea de Patentes señalan dos ejemplos de generalización excesiva:

a) Cuando se reivindica un procedimiento para el tratamiento de toda clase de plantas consistente en someterlas a un enfriamiento súbito del que resultan ciertos efectos, siendo así que el procedimiento descrito en la descripción no es aplicable más que a una sola especie vegetal y que existen razones para pensar que no es aplicable a todas las plantas, dadas las conocidas diferencias que existen entre las diversas especies.

b) Cuando se reivindica un método determinado de tratamiento de «moldeados de resina sintética», destinado a provocar ciertas modificaciones de sus propiedades físicas, siendo así que tơdos los ejemplos descritos se refie- 
ren a resinas termoplásticas y que el método no parece utilizable para resinas termoendurecibles.

MOUSSERON señala otros ejemplos.

Así, una reivindicación para proteger un procedimiento que permite actuar sobre sustancias por una energía de alta frecuencia, si la descripción expone solamente un procedimiento de eliminación de residuos de un gas por una energía de alta frecuencias.

O una reivindicación general de fijación, cuando la descripción se refiere sólo a la fijación por pegamento encolado.

En el procedimiento de concesión, la falta de fundamentación de las reivindicaciones en la descripción se puede subsanar, en general, modificando las reivindicaciones, para adaptarlas a la descripción. Y eso mismo puede hacerse, en determinados casos, después de la concesión. A este tema se hará referencia más adelante.

Pero también puede haber lugar a una actuación distinta, esto es, modificando la descripción para incluir en ella un elemento que aparece claramente expuesto en las reivindicaciones presentadas y que no contradice lo expuesto en la descripcion.

Lo que hay que evitar, sin embargo, es que se pretenda completar la descripción en base a elementos de las reivindicaciones que no aparecen claros en ellas o que no encajan en la descripción tal como se presentó originariamente.

Se trata de evitar, en definitiva, que se presente una solicitud con una descripción insuficiente, tal vez porque la invención no estaba terminada o perfeccionada, y se pretenda cambiar después la descripción para incorporar elementos importantes que antes no aparecían en ella.

\section{Unidad de invención}

\section{a) Función del requisito de unidad de invención}

Para comprender el requisito de unidad de invención de las solicitudes de 
patente, es imprescindible partir de la finalidad básica que cumplen las patentes, que no es otra que la de difundir el conocimiento de las invenciones, de manera que los terceros puedan conocer no sólo su existencia, sino también la manera de ejecutarlas. Por eso se ofrece a los inventores la posibilidad de obtener el derecho exclusivo de explotación inherente a la concesión de la patente; para estimularles a dar a conocer sus invenciones de manera que los técnicos en la materia puedan ponerlas en ejecución.

Para cumplir esa finalidad es imprescindible que en la solicitud se haga una descripción que permita a los técnicos normales ejecutar la invención, y es imprescindible, además, que esa descripción se haga accesible al público.

Pero de nada serviría la posibilidad que deben tener los terceros de conocer la descripción, si las patentes no estuvieran adecuadamente clasificadas.

Es obvio que para que la publicidad y difusión de las invenciones patentadas sea eficaz, las patentes en las que figuran las descripciones correspondientes deben estar bien clasificadas. De manera que quien esté interesado en conocer las invenciones de un sector tecnológico determinado sepa que podrá hacerlo consultando las patentes incluidas en la clase o clases correspondientes a ese campo de la tecnología. Si las patentes no estuvieran clasificadas en atención al área tecnológica a que se refieren las invenciones patentadas, la consulta de las descripciones de tales invenciones será prácticamente imposible.

Por ello, se clasifican las patentes y existe el Arreglo de Estrasburgo relativo a la Clasificación Internacional de Patentes, hecho en Estrasburgo el 24 de marzo de 1971 y modificado en 1979.

El requisito de la unidad de invención cumple, por tanto, una función primordial: la de permitir una correcta clasificación de las patentes y hacer, por tanto, posible la difusión pública de las invenciones patentadas.

Si una misma patente pudiera tener por objeto invenciones totalmente distintas, pertenecientes a áreas tecnológicas sin ninguna relación entre sí, no sería posible clasificar correctamente la patente y alguna de las invencio- 
nes protegidas sería, de hecho, de imposible conocimiento por los terceros, que no podrían saber dónde estaba clasificada la patente correspondiente.

Por tanto, el requisito de la unidad de invención es ante todo fundamental para permitir la necesaria difusión pública de las invenciones patentadas.

Pero cumple, además, otra función recaudatoria, al hacer que se paguen tasas por cada invención patentada. Si no existiera el requisito de la unidad de invención sería fácil proteger diversas invenciones por medio de una única patente y pagando solamente las tasas que correspondan a la misma.

No cabe ignorar, por otra parte, que la exigencia de la unidad de invención tiene una trascendencia adicional con referencia a los sistemas de concesión de patentes con informe sobre el estado de la técnica o examen previo de la novedad y actividad inventiva.

En tales sistemas la falta de unidad de invención aumenta el coste de elaboración del informe, al tener que investigar los documentos de clases distintas, razón por la cual si existe pluralidad de invenciones deben pagarse tasas adicionales.

Y, desde otro punto de vista, durante el procedimiento de búsqueda de anterioridades o de examen previo, la Oficina de Patentes puede constatar la falta de unidad de invención y exigir la división de la solicitud de patente en varias solicitudes distintas. Ante un requerimiento de esa índole, a menudo el solicitante lo que hace es renunciar a alguna reivindicación, que es la que incumple la exigencia de la unidad de invención.

No debe pensarse, sin embargo, que la exigencia del pago de tasas adicionales vaya siempre acompañada, por parte de la Oficina de Patentes, de la imposición de que se divida la solicitud.

Por supuesto, en los sistemas de concesión que no incluyen la realización de un informe sobre el estado de la técnica o de un examen previo de novedad y actividad inventiva, existen muchas menos posibilidades de que la Oficina de Patentes detecte la falta de unidad de invención. Por ello tiene sentido dentro de tales sistemas, que la falta de unidad de invención de las 
patentes concedidas constituya causa de nulidad de la patente. Así se establecía, por ejemplo, en el derogado Estatuto español sobre Propiedad Industrial, de 26 de julio de 1929 (art. 57 en relación con el art. $115, \mathrm{n}^{\circ} 5^{\circ}$ ).

\section{b. Pluralidad de reivindicaciones y unidad de invención}

Como ya se ha expuesto anteriormente, las reivindicaciones definen las invenciones cuya protección se «reivindica» en la patente. $Y$ ocurre que no sólo es posible, sino que es normal, que en una misma patente se incluya una pluralidad de reivindicaciones.

Pues bien, ¿cómo se compatibiliza la pluralidad de reivindicaciones de una patente con la exigencia de unidad de invención?

Por supuesto que la unidad de invención existe cuando la patente incluye una única reivindicación en la que se define una regla técnica determinada.

Pero la invención, aun siendo única, puede admitir desarrollos concretos diferentes, que den lugar a reivindiciones dependientes; dependientes por ser desarrollo de la misma invención.

Ahora bien, el simple hecho de que una reivindicación se presente como dependiente no significa, por ese solo criterio formal, que se respete la unidad de invención. Habrá que determinar si la manifestación formal de la dependencia se corresponde realmente con la dependencia entre los objetos de las distintas reivindicaciones.

Por otra parte, hay que señalar también que aunque existan diversas reivindicaciones independientes, pueden cumplir con la exigencia de la unidad de invención, si todas ellas «integran un único concepto inventivo general» (art. 80 CPE y regla 13 PCT).

Para determinar si las reivindicaciones «integran un único concepto inventivo general», cabe seguir diversos criterios.

Un posible criterio a tener en cuenta es el de los documentalistas en- 
cargados de elaborar el informe sobre el estado de la técnica, atendiendo por lo tanto a las clases de documentos que deben ser consultados.

También debe considerarse si todas las reglas reivindicadas sirven para solucionar el mismo problema técnico.

Cuando el problema es resuelto por primer vez, puede considerarse que medios muy distintos reivindicados a tal efecto integran el mismo concepto inventivo general. Pero si el problema técnico ya había sido resuelto anteriormente, entonces debe aplicarse un criterio más restrictivo sobre la unidad de invención de las reivindicaciones referidas a distintas reglas que constituyen nuevas soluciones.

Igualmente podrá entenderse que existe unidad de invención, cuando las distintas reivindicaciones se refieren a reglas que sirven para satisfacer una misma necesidad aplicando medios que pertenecen al mismo sector de la técnica.

Son ejemplos habituales de pluralidad de reivindicaciones, que cumplen con la unidad de invención, las que se refieren a un producto, el procedimiento para su obtención, el aparato para la ejecución del procedimiento y la aplicación del producto, que responden todas ellas al mismo concepto inventivo general.

También responderán a ese mismo concepto inventivo general las reivindicaciones que tienen por objeto el producto final y las que se refieren a los productos intermedios. Así lo declaro, por ejemplo, la Cámara de Recursos Técnicos de la Oficina Europea de Patentes en resolución de 25 de abril de 1982 (Caso «Copolycarbonatos»).

\section{Requisitos de forma}

\section{Redacción}

La reivindicación debe delimitar de forma clara y concisa la invención que se reivindica, expresando todas las características técnicas que sean necesarias y suficientes para definirla. 
Y la redacción de cada reivindicación debe ser autónoma, de manera que pueda ser entendida por sí misma, sin necesidad de acudir a otros documentos. Obsérvese que la exigencia de que la reivindicación pueda ser «entendida» por sí misma, no significa que por su sola redacción pueda ejecutarse la invención reivindicada.

Por lo que a la estructura se refiere, la regla 29 del Reglamento CPE dispone que toda reivindicación debe organizarse, en la medida de lo posible, en dos partes.

Una primera parte que contenga un preámbulo, designando el objeto de la invención, esto es, el campo técnico general -que forma parte del estado de la técnica - al que se refiere la invención, incluyendo las características técnicas necesarias para la definición de los elementos reivindicados, excepto aquellas que, aunque no se incluyan, son obvias para el experto en la materia.

Y una segunda parte caracterizante, donde se mencionan las características de la invención, que vienen a encuadrarse -completándolo o mejorándolo- en la parte del estado de la técnica expresada en el preámbulo.

Esta segunda parte comienza con las palabras «caracterizado por...».

Por ejemplo, si la invención se refiere a un aparato fotográfico y la actividad inventiva se relaciona con el obturador, la reivindicación podría tener como preámbulo: «aparato fotográfico con un obturador focal plano», y seguiría: «caracterizado por...», incluyendo a continuación las características técnicas de la invención.

En la discusión con los examinadores puede ocurrir que ciertas características que figuran en la segunda parte de la reivindicación hayan de pasarse a la primera, por considerar que ya estaban en el estado de la técnica. Por eso se dice que cuanto más abajo se pone la frase «caracterizado por...», más se limita la invención reivindicada.

Hay que señalar que, en ocasiones, no es necesaria la división de la reivindicación en dos partes; por ejemplo, cuando se reivindican sustancias químicas por su fórmula. En las reivindicaciones, si bien pueden figurar fórmulas, no pueden figurar dibujos. 
En la parte caracterizante hay que evitar que se pretenda caracterizar la invención por el resultado perseguido. Ejemplo: cenicero que por su forma y proporciones sirve para apagar automáticamente las colillas.

\section{Terminología}

Las reivindicaciones están destinadas a ser comprendidas por el experto normal en la materia. Por ello debe utilizarse la terminología que sea de uso normal dentro del sector de la técnica de que se trate. Y si se utiliza una terminología distinta a la habitual en ese sector de la técnica, entonces el significado de los términos empleados debe resultar del texto de la propia reivindicación.

Debe evitarse las ambigüedades y los términos imprecisos («aproximadamente», «alrededor de», «ancho», «fino»...), salvo que por el contexto sean claros para un experto en la materia.

Y debe evitarse, igualmente, que por la utilización de términos abstractos (la «esencia de la invención», el «espíritu de la invención») se pretenda extender de forma imprecisa el objeto protegido por la reivindicación.

\section{Relación con otros documentos de la solicitud}

Las reivindicaciones han de tener autonomía frente a los restantes documentos de la solicitud de patente, de manera que puedan ser entendidas por sí mismas.

No son, pues, admisibles reivindicaciones fundadas en referencias a la descripción o a los dibujos, salvo en casos excepcionales de absoluta necesidad.

Esa necesidad existiría si la forma particular expresada en los dibujos es esencial o si los productos químicos sólo pueden definirse por gráficos o diagramas.

Se pueden incluir en las reivindicaciones referencias a los dibujos, pero como complementarias para facilitar la interpretación de aquéllas, no 
como sustitutivas de la definición. Por ejemplo, pueden ponerse números como referencia a los dibujos, para facilitar la interpretación de la reivindicación.

Naturalmente, las reivindicaciones deben fundamentarse en la descripción, y la descripción y los dibujos sirven a su vez para interpretar las rei-. vindicaciones, según se verá más adelante.

\section{Categorías de reivindicaciones según el objeto reivindicado}

Se distinguen diversas clases de invenciones, y siendo la invención el objeto de cada reivindicación, pueden distinguirse diversas categorías de reivindicaciones según su objeto.

Y la clase o categoría a que pertenezca la reivindicación es importante, por cuanto el tipo de invención que constituye el objeto de aquélla será esencial para enjuiciar su validez y delimitar su ámbito de protección.

Se distinguen tradicionalmente las invenciones de producto, de procedimiento, de aplicación del procedimiento, y de uso, aunque se señala en las directivas para el examen de la Oficina Europea de Patentes que pueden reducirse a dos las clases de reivindicaciones: las que se refieren a la definición de un objeto con entidad física (producto) y las que se refieren a la descripción de una actividad (procedimiento). En tal sentido, las invenciones de aplicación y de uso serían invenciones de procedimiento.

\section{Reivindicaciones de producto}

Las reivindicaciones de producto tienen por objeto la definición de un objeto con entidad física; puede ser una sustancia, una composición, un objeto, un artículo, un elemento de una máquina, una máquina, un aparato, un conjunto de aparatos funcionando conjuntamente.

Ejemplos:

— «Un mecanisco de dirección en el que se integra un circuito de reacción automática». 
— «Un traje tejido que comprende...»

— «Un insecticida constituido por...»

- «Un sistema de telecomunicaciones que comprende diversas estaciones emisoras y receptoras».

En las reivindicaciones de producto, la patentabilidad se juzga sobre el producto, considerado en sí mismo, prescindiendo del procedimiento de fabricación o su uso.

Y del mismo modo, la protección de la patente comprende la explotación del producto mismo, cualquiera que sea su procedimiento de fabricación y su uso. El derecho exclusivo comprende, por tanto, tanto la fabricación del producto, como su uso y comercialización.

\section{Reivindicaciones de procedimiento}

El procedimiento describe una actividad a realizar sobre una materia, sobre una energía, sobre otros procedimientos, sobre materias vivas. do.

Y la patentabilidad se juzga sobre el procedimiento mismo reivindica-

El derecho exclusivo que atribuye una reivindicación de procedimiento comprende la ejecución de la actividad en que consiste el procedimiento, y comprende también el resultado directamente obtenido por la ejecución de tal actividad, tenga o no ese resultado entidad física autónoma (sustancia química, barnizado o pintado de coche).

Pero para la extensión del derecho exclusivo al producto directamente obtenido por el procedimiento, será necesario probar que el producto ha sido efectivamente obtenido por el procedimiento, porque si hubiera sido obtenido por un procedimiento distinto, entonces no caería su explotación bajo la exclusiva de la patente.

\section{Otras reivindicaciones}

Las invenciones de nuevas aplicaciones o nuevos usos se refieren a supues- 
tos en que la innovación consiste en la nueva aplicación o el nuevo uso, que se describe, de procedimientos o productos ya conocidos. A menudo ocurre, por ello, que tales invenciones son dependientes de las correspondientes al procedimiento o productos anteriores, y si tales invenciones anteriores están patentadas, no podrá explotarse la nueva aplicación o el nuevo uso sin el consentimiento del titular de la patente que protege la invención principal.

Esa dependencia entre las invenciones explica que, a menudo, una misma invención haya de protegerse mediante reivindicaciones distintas, y que en ocasiones se produzcan solapamientos entre los derechos exclusivos correspondientes a las distintas reivindicaciones. Así, por ejemplo, la utilización de un aparato reivindicado puede constituir la ejecución de un procedimiento objeto de otra reivindicación, cuando el aparato se ha concebido precisamente para ejecutar ese procedimiento.

\section{Pluralidad de reivindicaciones}

Una misma idea inventiva puede dar lugar a invenciones diversas, que habrán de ser objeto de reivindicaciones independientes, y también puede ocurrir que una misma invención puede dar lugar a modos diversos de desarrollo, que deban ser cubiertos también por distintas reivindicaciones.

Por ello es lo normal que en una misma patente se incluyan varias reivindicaciones, razón por la cual ya se ha visto que se plantea el problema de la unidad de invención.

Ahora bien, cuando una patente comprende varias reivindicaciones, éstas pueden ser independientes o dependientes de otra u otras.

Reivindicaciones independientes, como su nombre indica, son aquellas que tienen total autonomía de las demás.

Por el contrario, son dependientes aquellas que incluyen todas las características de otra reivindicación.

El hecho de que una reivindicación haga referencia a otra no significa que sea dependiente de ella. 
Así, por ejemplo, una reivindicación para un «dispositivo para la ejecución del procedimiento de la reivindicación $\mathrm{x}$ » o para un «procedimiento de fabricación del producto de la reivindicación y».

Cada reivindicación independiente debe considerarse autónomamente, como si fuera la única de la patente, y juzgarse su patentabilidad aisladamente.

Por el contrario, la reivindicación dependiente va vinculada a la anterior de la que depende, pero no se excluye que pueda contener una invención autónoma.

Así ocurriría, por ejemplo, si se reivindica un modo de realización de un aparato con un material determinado, cuando el material es en sí mismo una invención; o un modo de ejecución de un procedimiento químico con una sustancia determinada que constituye en sí misma una invención.

En relación con las reivindicaciones dependientes se plantea el problema de su posible subsistencia en el caso de que desaparezca, por nulidad o por cualquier otra causa, la reivindicación principal con la que existe el nexo de dependencia.

Podría admitirse esa subsistencia si la reivindicación dependiente contiene algún elemento inventivo propio; pero el principio general es que la nulidad de la reivindicación principal arrastra a las dependientes.

La dependencia puede referirse a los modos de realización de la invención objeto de la reivindicación principal, y también a diversas aplicaciones o usos de la misma; y puede tener por objeto simples desarrollos de la reivindicación principal o aportar elementos inventivos propios.

Una reivindicación dependiente puede tener también otras dependientes de ella.

La pluralidad de reivindicaciones plantea la necesidad de limitar el número máximo de ellas que pueden incluirse en la misma patente; por ello, por ejemplo, en el sistema de concesión de las patentes europeas, deben pagarse tasas suplementarias por las reivindicaciones que excedan de diez (regla 31 del Reglamento). 


\section{Particularidades de reivindicaciones referentes a determinados tipos de invenciones}

\section{Invenciones químico-farmacéuticas}

Las invenciones que se producen en este campo pueden tener por objeto nuevas sustancias, nuevos procedimientos para obtención de sustancias ya conocidas, y nuevas aplicaciones.

Es, pues, posible reivindicar invenciones de todas esas clases, siempre que la legislación no prohíba expresamente patentar alguna de esas clases de invenciones, lo cual ocurre en algunos casos con las invenciones de nuevas sustancias químicas o farmacéuticas o de nuevas aplicaciones medicamentosas.

En relación con esta clase de invenciones se plantea un importante problema, por cuanto una misma invención puede referirse a familias con millones de compuestos.

En tales casos la dificultad consiste en que si se reivindica la invención solamente en relación con las sustancias que han sido ya objeto de experimentación, será fácil aplicar la invención a otras sustancias equivalentes, sin que esa aplicación caiga bajo el ámbito de protección del derecho exclusivo que corresponde a la patente.

Por el contrario, si se reivindica la invención indiscriminadamente para todos los compuestos integrantes de la misma familia, el riesgo es el de que pueda rechazarse la solicitud de la patente o declararse la nulidad de la patente concedida por falta de ejecutabilidad en algunos supuestos.

Parece que debería admitirse la generalización de la invención a toda la familia de compuestos, si a la vista de los experimentos realizados (entre ellos, de los compuestos marginales de la familia), y teniendo en cuenta las sustancias que reaccionan, es predecible con cierto grado de certeza, que la regla inventiva es válida para toda la familia.

Otro problema importante resulta de la prohibición de patentar las sustancias químicas y farmacéuticas que rige en algunos ordenamientos. 
Con esa prohibición se pretende que el titular de la patente de procedimiento no pueda impedir que otras personas, sin su autorización, obtengan la misma sustancia pero por un procedimiento distinto.

En tales casos, por tanto, los terceros pueden, o bien patentar otros procedimientos distintos para la obtención de la misma sustancia, sin que esas nuevas patentes sean dependientes de la anterior, o bien utilizar procedimientos distintos para la fabricación de la sustancia sin violar la patente.

Cuando está prohibido patentar la sustancia, se suscita con intensidad la cuestión de delimitar el ámbito de protección de las patentes de procedimiento para la obtención de sustancias. $\mathrm{Y}$ a tal efecto hay que considerar que el procedimiento queda integrado por la sustancia de partida, las reacciones u operaciones a realizar a partir de aquélla y la sustancia final. De manera que la sustancia final es parte del procedimiento y, por tanto, cae bajo la protección de la patente, siempre, naturalmente, que se haya obtenido a partir de la sustancia de partida y como resultado de las operaciones reivindicadas en el procedimiento.

Por ello se establece a menudo expresamente en las legislaciones, que la patente de procedimiento comprende en el derecho exclusivo el producto directamente obtenido por el procedimiento patentado (art. 64.2 CPE).

Ocurre, sin embargo, que cuando lo que se pretende es hacer valer el derecho exclusivo para impedir la comercialización de la sustancia que se obtiene por el procedimiento, es a menudo imposible probar, por el solo examen de la sustancia, el procedimiento a través del cual ha sido obtenida. $\mathrm{Y}$ al ser imposible esa prueba, que ha de aportar el titular de la patente, no puede hacerse valer el derecho exclusivo. Este problema es fundamental y se da especialmente cuando se pretende hacer valer la protección de la patente para impedir la comercialización de sustancias importadas desde el extranjero.

Por ello es importante la norma que existe en muchos ordenamientos (por ejemplo, art. 61.2 de la Ley española de patentes de 1986), según la cual se invierte en tales casos la carga de la prueba, al disponer que cuando a través de un procedimiento se obtiene una nueva sustancia, se presume, salvo prueba en contrario, que toda sustancia de las mismas características ha sido producida por el procedimiento patentado. 
Si esa inversión de la carga de la prueba existe, deberá ser el demandado, esto es, el tercero que comercializa la sustancia nueva, el que deba probar que ha sido obtenida por un procedimiento distinto al que está reivindicado y protegido por la patente del demandante.

Cabe señalar, además, que en los ordenamientos en que se prohíbe patentar las sustancias químicas o farmacéuticas, ocurre en ocasiones que verdaderas invenciones de producto, como ocurre por ejemplo con las mezclas, se intentan enmascarar como invenciones de procedimiento, reivindicándolas como tales.

Por lo que se refiere a la prohibición de patentar las sustancias farmacéuticas o medicamentosas, la prohibición impide también que puedan patentarse $-\mathrm{y}$, por consiguiente, reivindicarse- las invenciones de uso 0 aplicación medicamentosa, tanto las que se refieren a un primer uso farmacéutico, como a nuevas aplicaciones sucesivas de esa misma naturaleza, para tratamientos médicos diversos.

\section{Invenciones microbiológicas}

La patentabilidad de las invenciones microbiologicas plantea también problemas específicos.

El primero consiste en que a menudo es imposible definir los microorganismos a los que se refiere la invención. En tales casos será suficiente que en la reivindicación se mencione el microorganismo que haya sido depositado en una institución autorizada al efecto. Para facilitar ese depósito y dar los efectos mencionados, existe el Tratado de Budapest sobre reconocimiento internacional del depósito de microorganismos a los fines del procedimiento en materia de patentes, hecho en Budapest el 28 de abril de 1977.

En segundo lugar, la reivindicación del procedimiento de obtención de los microorganismos no se adapta a las exigencias de protección del derecho exclusivo sobre éstos, tanto si se trata de un procedimiento de manipulación genética, como si consiste en un procedimiento de aislamiento de microorganismos que ya están en la naturaleza. La falta de adaptación resulta del hecho de que una vez producido o aislado el microorganismo, su 
explotación se hará, no mediante la ejecución del procedimiento, sino por la reproducción biológica del propio microorganismo.

Estos problemas explican que deban configurarse o desarrollarse normas especiales para la protección y reivindicación de esa clase de invenciones. Ése es el sentido que tiene la Propuesta de Directiva del Consejo de la Comunidad Europea relativa a la protección jurídica de las invenciones biotécnológicas (Propuesta modificada presentada por la Comisión al Consejo, tras las modificaciones introducidas por el Parlamento Europeo; vid. Diario Oficial de las Comunidades Europeas, $\mathrm{n}^{\circ} \mathrm{C} 305 / 160$, de 23.11.92).

\section{Las reivindicaciones y de la delimitación del ám- bito de protección de la patente: interpretación de las reivindicaciones}

Como ya se ha dicho, es función de las reivindicaciones delimitar el objeto sobre el que se concede al titular la exclusiva de explotación que atribuye la patente.

En alguna ocasión, sin embargo, el ámbito objetivo de protección puede exceder por disposición legal del contenido de la reivindicación. Así ocurre, cuando la ley extiende el derecho exclusivo en las patentes de procedimiento al producto directamente obtenido por procedimiento patentado.

Naturalmente, para determinar el objeto reivindicado es normalmente imprescindible proceder a la interpretación de la propia reivindicación.

$Y$ el interés del titular en una interpretación extensiva o restrictiva de la reivindicación variará según se trate de juzgar sobre la validez o sobre una violación de la misma. De ahí la difícil situación en que se encuentra el titular cuando ha demandado a un tercero por violación de su patente y el tercero ha presentado demanda reconvencional pidiendo la nulidad de la patente o de alguna reivindicación.

La interpretación debe ser hecha por un experto normal en el campo de la técnica de que se trate. Ese experto normal no podrá ser un obrero manual, ni un especialista de primera fila. Pero puede ocurrir que en el mo- 
mento de la solicitud en el campo técnico en cuestión sólo existieran técnicos altamente especializados, y será preciso acudir a ellos.

Evidentemente cuando una reivindicación es clara, no debe pretenderse limitar su ámbito introduciendo frases o palabras que no están en ella.

Pero tradicionalmente se han seguido dos criterios sobre el significado e interpretación de las reivindicaciones.

Por una parte, el criterio que puede denominarse de «poste de vallado o de cerca», que es el criterio anglosajón.

Y por otro lado, el criterio que puede llamarse "poste señalizador», según el cual queda comprendido en la reivindicación lo que con ayuda de la descripción y los dibujos podría deducir de la reivindicación un experto normal, esto es, la idea inventiva general. Podría decirse que la reivindicación actuaría, dentro de este criterio, como el poste señalizador de una veta de mineral, que diera derecho a todo el mineral que pudiera extraerse de ella.

Cabe decir que a nivel europeo ha prevalecido el criterio del «poste de vallado o de cerca». Es decir, que lo protegido es lo reivindicado; pero ese criterio se acerca algo al otro, en cuanto que la delimitación de lo reivindicado debe hacerse interpretando la reivindicación, especialmente en los casos de ambiguiedad, con ayuda de la descripción y los dibujos (art. $69 \mathrm{CPE}$ ). Ahora bien, lo que no puede hacerse es completar o ampliar el contenido de la reivindicación acudiendo a la descripción o los dibujos.

La interpretación debe hacerse por un experto normal para que caigan bajo la protección los equivalentes que cualquier experto conoce a los elementos de la regla reivindicada.

\section{La mofificación de las reivindicaciones y sus efectos}

Siendo las reivindicaciones un elemento esencial de la patente, es obvio que 
no hay solicitud de patente mientras no se presente una solicitud con una reivindicación al menos. Sin reivindicación no hay fecha de presentación.

$\mathrm{Y}$ al ser la fecha de presentación la que sirve para juzgar sobre la patentabilidad, es obvio que la posterior modificación de las reivindicaciones afecta gravemente a los terceros, pues significaría poder dar una fecha de presentación o prioridad antedatada y ficticia a las reivindicaciones, $y$, por consiguiente, a la solicitud de la patente.

La seguridad jurídica exige que no se modifiquen las reivindicaciones, y por ello deben regularse muy estrictamente los supuestos en que tal modificación es posible, así como los efectos de la misma.

Las modificaciones pueden tener lugar durante el procedimiento de concesión y después de que la patente ha sido concedida.

En cualquier caso, el principio general consiste en que no puede beneficiarse de la fecha de prioridad de una reivindicación lo que no estaba incluido en ella en la fecha de presentación de la misma.

Para el tratamiento de las modificaciones hay que distinguir la posibilidad y régimen de las mismas, según que se pretendan introducir antes o después de la concesión de la patente.

\section{Antes de la concesión de la patente}

Antes de la concesión de la patente la modificación puede hacerse para subsanar errores, y, especialmente en los procedimientos con informe sobre el estado de la técnica o examen previo, para adaptar la reivindicación a las anteriores que aparecen en el informe o para llegar a un acuerdo con el examinador.

Y pueden seguirse dos sistemas sobre los efectos a atribuir a tales modificaciones.

Un primer sistema consistiría en que la fecha de prioridad de la parte nueva de la reivindicación debe ser la de la fecha efectiva en que se hizo la 
modificación de la misma y no la fecha de presentación de la solicitud de patente.

Otro sistema distinto consiste en considerar que es causa de nulidad la extensión del contenido de la reivindicación como consecuencia de una modificación de la misma (art. $123 \mathrm{CPE}$ ).

Otra modificación puede consistir en la necesaria división de la solicitud por falta de unidad de invención.

\section{Después de la concesión de la patente}

Después de la concesión de la patente la modificación de la reivindicación debe ser absolutamente excepcional y podría producirse por renuncia del titular o por declaración judicial de nulidad que afecten sólo a una parte de la reivindicación.

Hay ordenamientos en los que la modificación parcial de una reivindicación de una patente ya concedida no es posible. Así, la Ley española de patentes de 1986, en su artículo 112.2, sólo permite que se declare la nulidad de reivindicaciones completas.

En otros sistemas, se admite, sin embargo, que se modifiquen las reivindicaciones, dándoles una nueva redacción, tal como establece el Convenio de Luxemburgo relativo a la Patente Europea para el Mercado Común, de 15 de diciembre de 1975 (Convenio sobre la patente comunitaria que todavía no está en vigor), que permite que el titular de la patente limite el objeto de la misma, modificando las reivindicaciones, la descripción o los dibujos, previa solicitud presentada al efecto (art. 51.1).

No cabe ignorar que este segundo sistema es mucho más complejo que el primero, por las grandes dificultades que suscita el control de la nueva redacción, para evitar que altere el contenido de la invención reivindicada más allá de lo exigido por la renuncia o por la declaración de nulidad. 


\section{Bibliografía}

BENKARD, Georg

1981 Patentgesetz. Gebrauchsmustergesetz. $7^{\text {a }}$ ed. Verlag C. H. Beck, München.

CORNISH, W. R.

1981 Intellectual Property. Sweet \& Maxwell, London, y $2^{\mathrm{a}}$ ed., 1989 London.

CHARTERED INSTITUTE OF PATENTS AGENTS: C. I. P. A.

1990 Guide to the Patents Act. $3^{\text {a }}$ ed. Sweet \& Maxwell, London.

EUROPEAN PATENT OFFICE

1992 Guidelines for examination in the European Patent Office. January.

GODENHIELM, Berndt

1989 «Quelques réflexions sur l'interpretation des revendications des brevets d'invention», Mélanges Joseph Voyame, diffusion Payot, Lausanne.

KELLY, Patrick D.:

1989

«Claim Language in Biotechnology Patents», in Biotechnology, Vol. 7, february.

KRASSER, Rudolf

1986 Lehrbuch des Patentrechts begründet von Wolfgang Bernhardt. $4^{\text {a }}$ ed., C. H. Beck, München.

MEINHARDT, Peter

1971 Inventions, Patents \& Trade Marks, A. Goner Press Limited, London. 
MILLER, Arthur R. y DAVIS, Michael H.

1983 Intellectual Property. West Publishing Co., St. Paul, Minn.

MOUSSERON, Jean Marc

1984 Traité des Brevets. L'obtention des brevets. Librairies Techniques, Paris.

PHELIP, Bruno

1989 Brevets d'invention, $3^{\text {a }}$ ed., J. Delmus et Cie, Paris.

SCHULTE, Reiner

$1987 \quad$ Patentgesetz. $4^{\mathrm{a}}$ ed. Heymanns, Köln.

TROLLER, Alois

Immaterialgüterrecht. 2 tomos. $3^{\mathrm{a}}$ ed., Helbing \& Lichtenhahn, Basel/Frankfurt am Main. 